\title{
X-ray induced mass loss effects on exoplanets orbiting dM stars
}

\author{
T. Penz and G. Micela
}

\author{
INAF - Osservatorio Astronomico di Palermo, Piazza del Parlamento 1, 90134 Palermo, Italy \\ e-mail: [tpenz;giusi]@astropa.inaf.it
}

Received 18 October 2007 / Accepted 7 November 2007

\section{ABSTRACT}

\begin{abstract}
Aims. We study the influence of the X-ray luminosity distribution of dM stars on mass loss from planets on close-in orbits. Methods. Using the X-ray luminosity of the Pleiades, the Hyades, and field dM stars, we construct a scaling law for the radiation environment of dM stars for ages between 0.1 and $10 \mathrm{Gyr}$. An energy-limited escape approach is used to calculate the influence of thermal mass loss on planetary distribution functions.

Results. We show that the X-ray luminosity distribution of nearby dM stars can be described by using a scaling law derived from observations of open clusters with a given age. It is shown that the X-ray flux from dM stars is significantly less than the flux from dG stars for a given orbital distance. Therefore, loss processes have less of an impact on the mass evolution of planets orbiting $\mathrm{dM}$ stars. We found that the mass loss is negligible for hydrogen-rich Jupiter-mass planets at orbits $>0.02$ AU, while Neptune-mass planets are influenced up to $0.05 \mathrm{AU}$. At orbits of $0.02 \mathrm{AU}$, Roche lobe effects are also having a strong impact on the mass-loss evolution. Because of the low mass of dM stars, Roche lobe effects are less effective for loss processes at planets orbiting these stars. Finally, if we use only the X-ray luminosity of their host stars for the energy input to the atmosphere, we obtain a lower limit for the mass loss of GJ876d and GJ674b. This does not allow us to conclude whether they are remnants of eroded Jupiter-mass planets.
\end{abstract}

Key words. X-rays: stars - stars: luminosity function, mass function - planets and satellites: general - stars: late-type stars: planetary systems

\section{Introduction}

A large fraction of the stars in our galactic neighborhood are $\mathrm{M}$ dwarf stars with masses between 0.1 and $0.5 M_{\odot}$. While their low luminosity makes it more difficult to obtain high signalto-noise observations, their low stellar mass and radius make it easier to detect low mass and small planets because of their higher contrast in radial velocity and transit photometric occultation depth. Observations indicate that $\mathrm{dM}$ stars might be a suitable environment for hosting planets, since accretion disks are rather common around young dwarf stars (e.g., Liu et al. 2004; Lada et al. 2006). From simulations it was found that Neptunemass planets might form more frequently around low-mass stars than Jupiter-mass planets, but the presence of the latter is not ruled out (Laughlin et al. 2004; Ida \& Lin 2005). Until now (October 2007), there are $6 \mathrm{M}$ dwarfs known to harbor planets. The planets orbiting GJ 436 (Butler et al. 2004), GJ 581 (Bonfils et al. 2005; Udry et al. 2007), and GJ 674 (Bonfils et al. 2007) have significantly lower masses than Jupiter, but there are also 4 Jupiter-mass companions found around M dwarfs, namely around GJ 876 (Marcy et al. 1998; Rivera et al. 2005), GJ 849 (Butler et al. 2006), and GJ 317 (Johnson et al. 2007). The triple system around GJ 876 (Rivera et al. 2005) harbors two Jupitermass planets and the Super-Earth GJ 876d. However, none of them is a close-in Hot Jupiter. From these observations it has been suggested that the frequency for hosting Jupiter-mass planets is 2-3 times greater for solar-mass stars than for $\mathrm{M}$ dwarfs (Butler et al. 2006; Johnson et al. 2007).

In this work we will discuss the influence of high-energy radiation from $\mathrm{dM}$ stars on atmospheric loss of planets in close-in orbits with masses ranging from $0.2 M_{\text {nep }}-5 M_{\text {jup }}$ as done for $\mathrm{dG}$ stars in Penz et al. (2008). We will take the evolution of activity during the main sequence life into account, and in particular we use the X-ray luminosity distribution function for $\mathrm{dM}$ stars from the Pleiades and the Hyades (Preibisch \& Feigelson 2005), which have a known age, to construct a scaling law for the temporal evolution of X-ray luminosity distribution function from 0.1-12 Gyr similar to what was done by Penz et al. (2008) for $\mathrm{dG}$ stars. One advantage of using the X-ray luminosity functions is that they allow us to also take the flare frequency into account. Indeed, the X-ray luminosity functions are obtained from a large sample of stars, assuming that they are ergodic systems, and the derived luminosity functions will automatically account for the time that a star spend flaring, except perhaps for the rarest events.

These X-ray luminosity distributions will be used as input for a modified energy-limited escape approach formula (Erkaev et al. 2007; Penz et al. 2008) to study mass-loss processes from exoplanets. This is a novel approach, because previous models investigating loss from planetary atmospheres (e.g., Lammer et al. 2003; Yelle 2004; Lecavelier des Etangs et al. 2004; Baraffe et al. 2004, 2005; Tian et al. 2005; Hubbard et al. 2006, 2007; Garcia Muñoz 2007) have focussed on solar-type G stars. Lecavelier des Etangs (2007) also included dM stars in his work, but does not take the luminosity distribution of these stars into account. The topic of the high-energy radiation impact of $\mathrm{dM}$ stars on planetary atmospheres is also discussed in Scalo et al. (2007) and Selsis et al. (2007) in a qualitative way, but they do not present detailed calculations of atmospheric escape. Here we present a detailed study on atmospheric loss caused by X-ray luminosity from $\mathrm{M}$ dwarf stars.

\section{Temporal evolution of $\mathbf{M}$ dwarf X-ray luminosity}

The Sun in Time program (Dorren \& Guinan 1994; Ribas et al. 2005) was established to investigate the radiation history of solar-type G stars. Similar programs were proposed for lower 
mass stars (Guinan et al. 2007) where a sample of $15 \mathrm{M}$ dwarf stars with various age should be used as proxies. However, such an approach suffers from large uncertainties due to the difficulties precisely dating $\mathrm{dM}$ stars. In particular, all the methods used (activity, kinematics, etc.) are statistical age indicators and so work for large samples. Therefore we are following another approach based on open clusters, which also has the advantage of taking into account the whole range of X-ray luminosity from $\mathrm{dM}$ stars, which is varying over more than one order-ofmagnitude for a given age (e.g., Schmitt et al. 1995; Preibisch \& Feigelson 2005). We are forced to work with X-ray data, since due to stellar absorption, it is not possible to achieve information about a large sample of stars in the EUV from 200-900 A. Since $\mathrm{X}$-rays play an important role in the heating of planetary atmospheres (Cecchi-Pestellini et al. 2006), we consider this to be a first step in understanding thermal escape processes from planets orbiting dM stars. Since our study does not include XUV radiation, the results can be considered as lower limits.

To investigate the temporal evolution of the X-ray luminosity, we consider data from stellar clusters with a given age, similar to Penz et al. (2008). In this case we may precisely date the stars (using the cluster age) and, at the same time, may account for the spread of the X-ray luminosity at a given age. We used the the Pleiades with an age of about $100 \mathrm{Myr}$ (Stauffer et al. 2005) and the Hyades with an age of about 600 Myr (Stauffer et al. 2005). The X-ray luminosity distributions for M dwarfs in these clusters can be found in Preibisch \& Feigelson (2005), who use data from Stelzer \& Neuhäuser (2001). The median values of the luminosity distributions are $L_{X}=10^{28.75} \mathrm{erg} / \mathrm{s}$ for the Pleiades and $L_{\mathrm{X}}=10^{28.15} \mathrm{erg} / \mathrm{s}$ for the Hyades (Fig. 1). Additionally, we need a distribution for older stars, where no clusters have been observed up to now for sensitivity reasons. Therefore we use Prox Cen as a proxy for older stars. Using astroseismology, the age of the triple system has been determined to be around $6 \mathrm{Gyr}$ (Eggenberger et al. 2004; Miglio \& Montalbán 2005). From the NEXXUS database (Schmitt \& Liefke 2004), the X-ray luminosity of Prox Cen is found to be $L_{X}=10^{26.81} \mathrm{erg} / \mathrm{s}$ outside of significant flares. In contrast to $\mathrm{dG}$ stars, the luminosity function for dM stars in the Pleiades and the Hyades cannot be fitted well by using a log-normal distribution (compare with Penz et al. 2008), so we use the observed distribution functions and inter/extrapolate from $0.1-12$ Gyr in order to achieve the temporal behavior of the X-ray luminosity under the assumption that the distribution function at the age of $6 \mathrm{Gyr}$ has a median value corresponding to the X-ray luminosity of Prox Cen and the same shape as for the Hyades. For the median value of the X-ray distribution function, a scaling law

$L_{X}= \begin{cases}0.17 L_{0} t^{-0.77} & t \leq 0.6 \mathrm{Gyr} \\ 0.13 L_{0} t^{-1.34} & t>0.6 \mathrm{Gyr}\end{cases}$

can be found, where $L_{0}$ is the mean log luminosity of the Pleiades $\left(10^{28.75} \mathrm{erg} / \mathrm{s}\right)$ and $t$ the age of the system in Gyr. In Fig. 2 the temporal evolution of $L_{X}$ is shown for dM stars and compared with the one for dG stars derived by Penz et al. (2008). Additionally, we show the X-ray luminosity given in Schmitt \& Liefke (2004) for the planet hosting stars GJ $876\left(10^{26.5} \mathrm{erg} / \mathrm{s}\right)$ and GJ $674\left(10^{27.6} \mathrm{erg} / \mathrm{s}\right)$. The bars indicate the uncertain age of the stars, which is between 0.1-1 Gyr for GJ 674 (Bonfils et al. 2007) and 6-10 Gyr for GJ 876 (Saffe et al. 2005).

The consistency of the scaling law (Eq. (1)) can be verified by reconstructing the observed X-ray luminosity function for nearby $\mathrm{dM}$ stars where a mixture of age is present, as given in Schmitt et al. (1995) assuming a constant stellar birth rate and

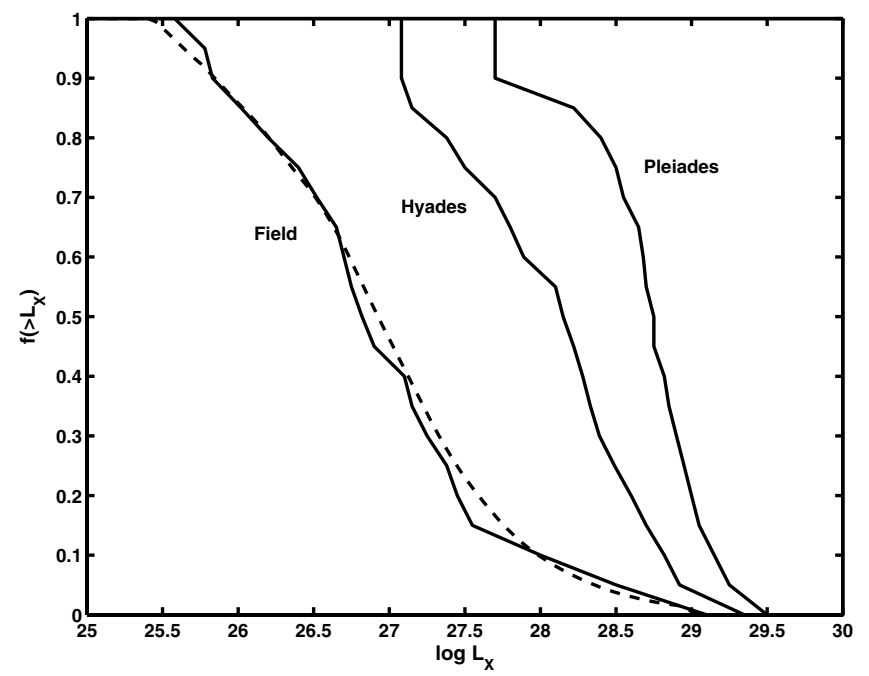

Fig. 1. Observed luminosity distribution functions for dM stars for the Pleiades and the Hyades (from Stelzer \& Neuhäuser 2001) and the field (from Schmitt et al. 1995). The dashed line shows the reconstructed luminosity distribution using the method described in the text.

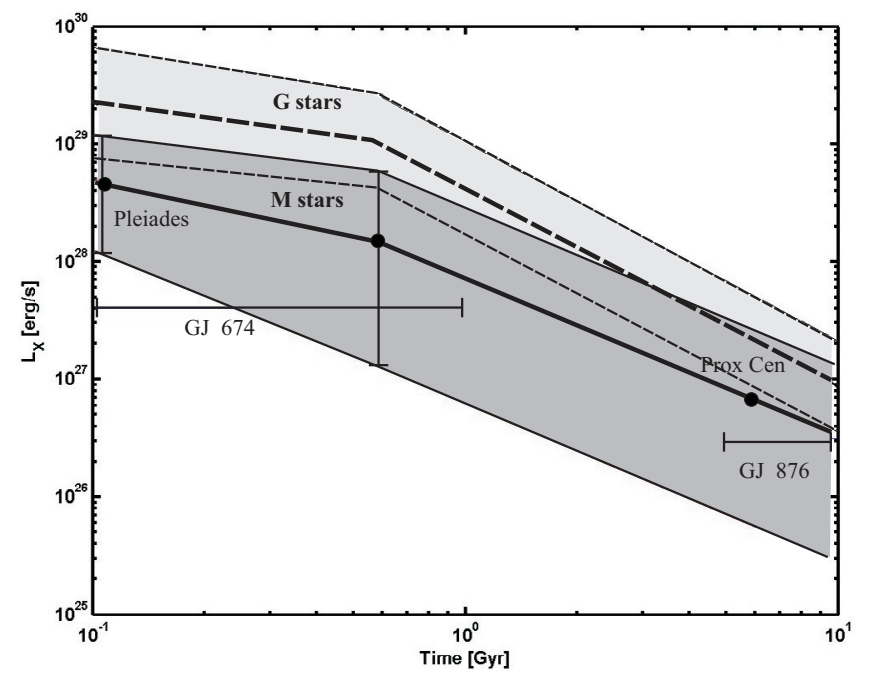

Fig. 2. The temporal evolution of $L_{\mathrm{X}}$ of $\mathrm{dM}$ stars (solid lines) and of $\mathrm{dG}$ stars (dashed lines). The thick lines give the median value, while the shaded areas represent the $1 \sigma$ equivalent of the observed luminosity distributions. The values for G stars are from Penz et al. (2008). The horizontal bars indicate the observed X-ray luminosity and age for GJ 876 and GJ 674.

a maximum age of 12 Gyr as in Penz et al. (2008). As can be seen from Fig. 1, a good agreement is found.

One can see that the X-ray luminosity of dM stars is several times lower than for $\mathrm{dG}$ stars, consistent with other studies (e.g., Preibisch \& Feigelson 2005). Interestingly, it can be seen that the upper $1 \sigma$ curve is flatter than the curve for the median value and the lower $1 \sigma$ curve. This most likely indicates a saturation effect at high luminosities as observed by Pizzolato et al. (2003). The saturation of coronal emissions implies a maximum value of $\log \left(L_{\mathrm{X}} / L_{\mathrm{bol}}\right) \approx-3$ (Vilhu \& Walter 1987), therefore the maximum X-ray luminosity that may be reached by a dM star is lower than the maximum value of dG stars. This implies that the saturation lasts a longer time in $\mathrm{dM}$ stars because of their lower bolometric luminosity. Some authors (Selsis et al. 2007; Scalo et al. 2007; Lammer et al. 2007) have discussed the temporal evolution of the ratio $L_{\mathrm{X}} / L_{\mathrm{bol}}$, which is higher for $\mathrm{dM}$ stars than 


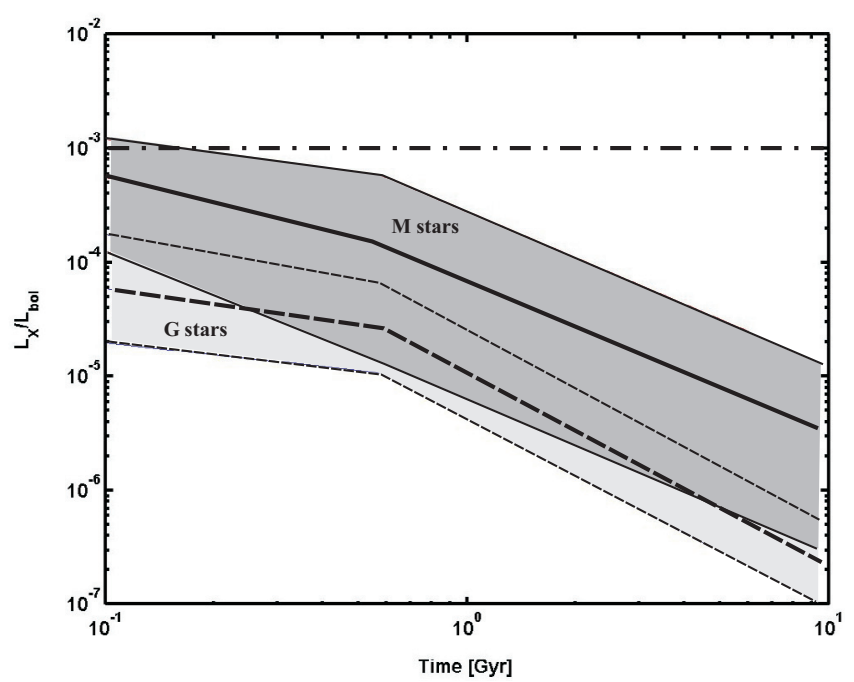

Fig. 3. Similar as Fig. 2, but for $L_{\mathrm{X}} / L_{\mathrm{bol}}$. The horizontal dashed-dotted line marks the saturation level of $10^{-3}$ (Vilhu \& Walter 1987).

for $\mathrm{dG}$ stars. They conclude that planets around $\mathrm{M}$ dwarfs might be exposed to higher radiation levels over a longer time period compared with dG stars. This is only true in the habitable zones of the respective stars, which are defined by $L_{\mathrm{bol}}$, thus $L_{\mathrm{X}} / L_{\mathrm{bol}}$ gives a useful estimate for comparing the $\mathrm{X}$-ray flux received by planets in the habitable zones of different stars. For comparison, we plot in Fig. 3 the ratio $L_{\mathrm{X}} / L_{\mathrm{bol}}{ }^{1}$, which is in qualitative agreement with the curves shown by Selsis et al. (2007) and Scalo et al. (2007). However, from $L_{\mathrm{X}} / L_{\text {bol }}$ we cannot directly derive the energy input for a planet at a given distance, so we use $L_{X}$ in the following, which is lower for $\mathrm{dM}$ stars than for $\mathrm{dG}$ stars at a given age and orbital distance.

\section{Atmospheric loss from gas planets orbiting dM stars}

Close-in exoplanets are subject to intense incident X-ray radiation, which can lead to high heating rates in their atmospheres, causing an expansion and atmospheric loss from the planet. Here we use a modified energy-limited escape approach, which has been developed by Erkaev et al. (2007) based on the work by Watson et al. (1981). It is a model for an escaping hydrogen atmosphere where the total incoming energy from stellar radiation is absorbed in a narrow region where the optical depth is unity. Erkaev et al. (2007) include the influence of Roche lobe effects, which are important at close-in distances. The same approach was also used in Penz et al. (2008), so we shall be brief in the following. The planetary mass loss is given as

$\frac{\mathrm{d} M}{\mathrm{~d} t}=\frac{4 \pi R_{\mathrm{pl}}^{3} F_{X}}{m M_{\mathrm{pl}} G K}$.

Here, $R_{\mathrm{pl}}$ and $M_{\mathrm{pl}}$ are the radius and mass of the planet, $m$ the mass of the hydrogen atom, $G$ the gravitational constant, $F_{X}$ the flux at the planets orbit derived from $L_{X}$, and $K$ takes Roche lobe effects into account (Erkaev et al. 2007).

${ }^{1}$ For dG stars we use the bolometric luminosity of the Sun $\left(L_{\mathrm{bol}}=\right.$ $\left.3.8 \times 10^{33} \mathrm{erg} / \mathrm{s}\right)$, while for $\mathrm{dM}$ stars we use $L_{\mathrm{bol}}=10^{32} \mathrm{erg} / \mathrm{s}$ (Cox 2001).

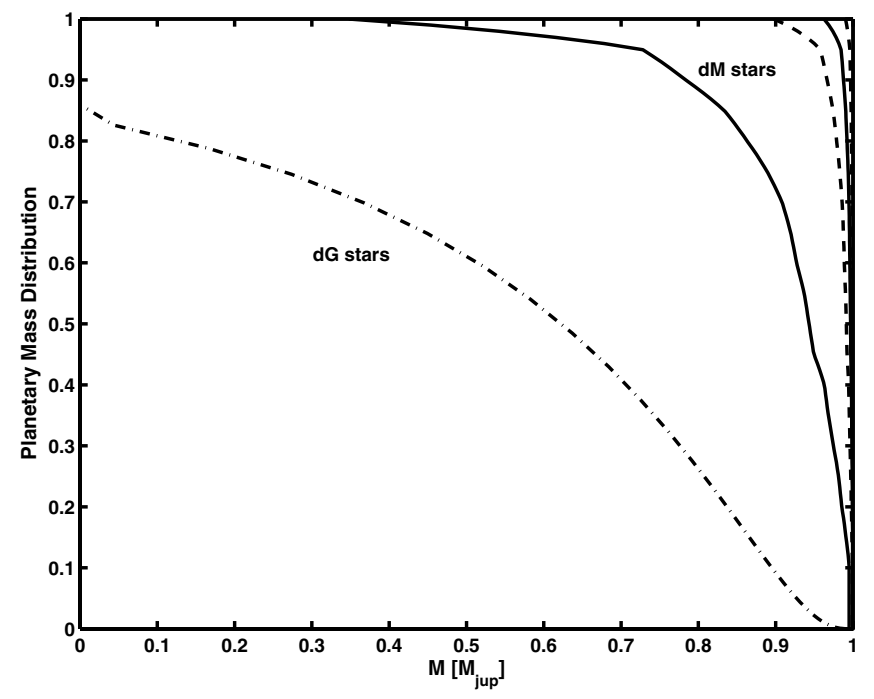

Fig. 4. Planetary mass distribution after 4 Gyr for an initial mass of $1 M_{\text {jup }}$, densities of 0.4 (solid lines) and 1.4 (dashed lines) $\mathrm{g} / \mathrm{cm}^{3}$ at $4 \mathrm{Gyr}$, and orbital distances of 0.02 and $0.05 \mathrm{AU}$ (starting from the lower curve). For comparison, the distribution function for low-density planets orbiting dG stars at $0.02 \mathrm{AU}$ is also shown (dashed-dotted line; taken from Penz et al. 2008).

\subsection{Final planetary mass distribution for a single initial mass}

For a given starting mass it is possible to trace the mass evolution of strongly irradiated gas planets by combining Eqs. (1) and (2). Since the X-ray luminosity is given in the form of a distribution function, the result of our calculations gives a mass distribution for the planetary mass. One important quantity that needs to be known is the density of the planet. Since the observed densities vary over a wide range, we use the temporal evolution of the planetary radius suggested by Lecavelier des Etangs (2007). The temporal evolution of the density following from the scaling law they have derived is given as $1 /\left(1+\beta t^{-0.3}\right)^{3}$, where $\beta=0.2$ for $M_{\mathrm{p}} \geq 0.3 M_{\text {jup }}$, and $\beta=0.3$ for $M_{\mathrm{p}} \approx 0.1 M_{\text {jup }}$. As shown in Penz et al. (2008), it is justified to stop the loss calculations after 4 Gyr, since the mass loss occurring after that time is negligible for $\mathrm{G}$ stars and will be even smaller for planets orbiting M stars as we have verified. In the following, we assume the mass of the host star to be $0.3 M_{\odot}$.

First, we apply this approach to an initial mass of one $M_{\text {jup }}$. We normalized the density scaling law to the observed density range from 0.4 to $1.4 \mathrm{~g} / \mathrm{cm}^{3}$ at an age of $4 \mathrm{Gyr}$. In these cases, the initial density at $0.1 \mathrm{Gyr}$ is 0.22 and $0.73 \mathrm{~g} / \mathrm{cm}^{3}$, respectively. Even at an orbital distance of $0.02 \mathrm{AU}$, none of the planets is completely evaporated. Two percent of the planets are losing slightly more than $50 \%$ of their mass for low-density planets, while nearly $90 \%$ of these planets can retain more than $80 \%$ of their mass (Fig. 4). For higher densities or larger orbital distances, virtually all planets retain their initial mass and are not affected by these mass-loss processes. For comparison, we also show the planetary mass distribution for low-density planets orbiting a G star at $0.02 \mathrm{AU}$ (Penz et al. 2008). One can clearly see that mass loss processes are much more efficient for planets around $\mathrm{dG}$ stars.

In Fig. 5 the planetary mass distribution is shown for planets with a starting mass of one $M_{\text {nep }}$ after 4 Gyr. The normalized density at 4 Gyr are 0.8 and $2 \mathrm{~g} / \mathrm{cm}^{3}$ (according to the density of GJ 436b (Gillon et al. 2007)), respectively. For the density evolution we use the scaling law of Lecavelier des Etangs (2007) 


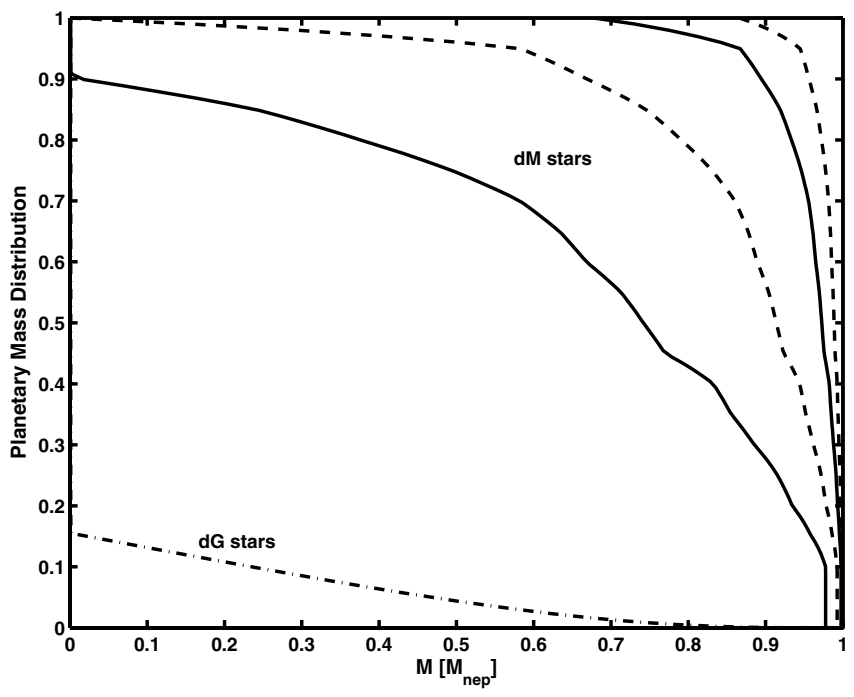

Fig. 5. Similar to Fig. 4 , but for an initial mass of $1 M_{\text {nep }}$, and densities of 0.8 (solid lines) and 2.0 (dashed-dotted lines) $\mathrm{g} / \mathrm{cm}^{3}$ after 4 Gyr. The dashed-dotted line shows the distribution function for a low-density Neptune orbiting a dG star at $0.02 \mathrm{AU}$.

valid for planets with $M_{\mathrm{p}} \approx 0.1 M_{\text {jup }}$. At an orbital distance of $0.02 \mathrm{AU}, 10 \%$ of the low-density and $1 \%$ of the high-density planets are evaporated or eroded to their rocky/icy cores. This implies that most of the planets at close-in orbits can retain an $\mathrm{H} / \mathrm{He}$ envelope over a lifetime, as indicated by observations (Demory et al. 2007) and modelling (Adams et al. 2007). Both, $42 \%$ (low density) and $80 \%$ (high density) of the planets can keep more than $80 \%$ of their initial mass. For a larger orbital distance of $0.05 \mathrm{AU}$, no planets are evaporated/eroded and only a few percent are subject to a loss of more than $20 \%$ in the lowdensity case. For comparison, the planetary mass distribution is again shown for a low-density planet at $0.02 \mathrm{AU}$ around a $\mathrm{G}$ star. One can see that evaporation effects are much stronger in this case.

\subsection{Roche lobe and radiation effects for $d M$ and $d G$ stars}

The approach used in the work by Penz et al. (2008) and in this paper also allows quantification of the influence of Roche lobe effects (Lecavelier des Etangs et al. 2004; Erkaev et al. 2007) and comparison with the influence of the host stars' radiation. In general, for planets with the same mass at the same orbit, Roche lobe effects are less pronounced at low-mass dM stars, since their mass is lower, shifting the L1 point away from the planet, resulting in lower mass loss.

To illustrate the effect, we assumed that stars with different masses have the same radiation output and evolution. Figure 6 shows the final planetary distribution function for a low-density Neptune-mass planet orbiting at $0.02 \mathrm{AU}$ for stellar masses ranging from $0.1-2 M_{\odot}$ around a star with the radiation environment of $\mathrm{dG}$ stars according to the scaling law given in Penz et al. (2008) and for dM stars according to the scaling law given in Eq. (1). With increasing stellar mass, the Roche lobe effect leads to higher losses when leaving all other parameters unchanged. For a mass of $0.1 M_{\odot}$, only $5 \%$ of the planets can be evaporated, while this number increases to $10 \%$ for $0.3 M_{\odot}$, and $20 \%$ for $1 M_{\odot}$ assuming the radiation of $\mathrm{dM}$ stars. For the radiation history of $\mathrm{dG}$ stars, the values ranging from $60 \%$ to $83 \%$ for $0.1 M_{\odot}$ to $1 M_{\odot}$. Comparing the curves for $0.3 M_{\odot}$ and the $\mathrm{dM}$ star radiation with the $1 M_{\odot}$ curve for dG stars shows

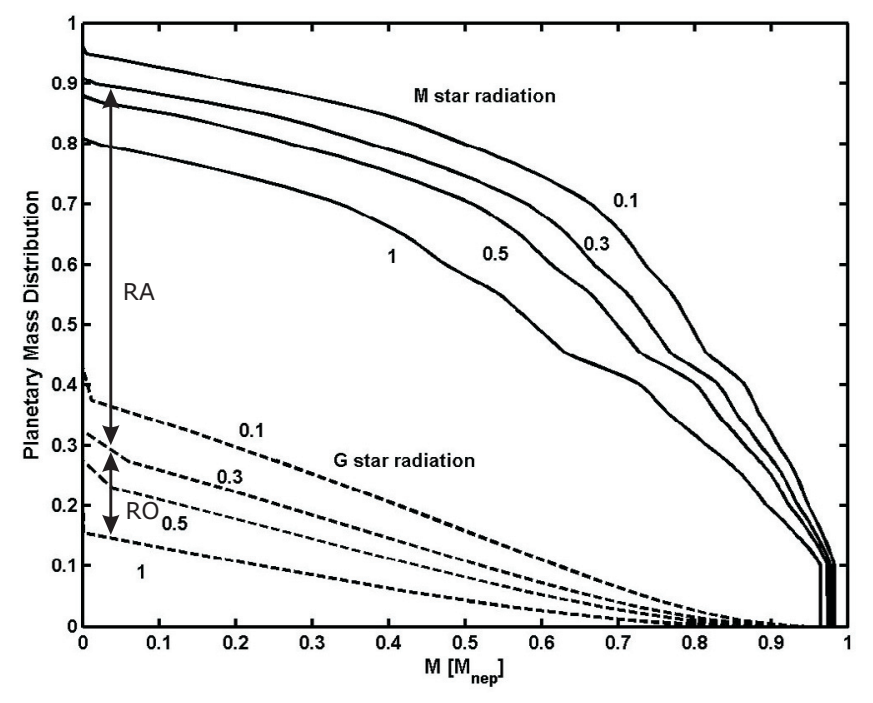

Fig. 6. Mass-loss effects for a planet with an initial mass of $1 M_{\text {nep }}$ and a final density of $0.8 \mathrm{~g} / \mathrm{cm}^{3}$ at $0.02 \mathrm{AU}$ after $4 \mathrm{Gyr}$ for the radiation environment of $\mathrm{dG}$ stars (dashed lines) and of $\mathrm{dM}$ stars (solid lines). The numbers indicate the stellar mass in $M_{\odot}$. RA indicates the range of radiation effects, RO indicates those for Roche lobe effects.

the cumulative effect of radiation and Roche lobe effects, which gives $10 \%$ evaporated planets for $\mathrm{dM}$ stars and $84 \%$ evaporated planets for dG stars. Figure 6 enables us to split up the different effects. The difference between the $0.3 M_{\odot}$ curves for $\mathrm{dG}$ and $\mathrm{dM}$ star radiation history gives the effect of the different radiation scaling laws between $\mathrm{dM}$ and $\mathrm{dG}$ stars (denoted RA), which is responsible for about $57 \%$ of the evaporated planets. The remaining $17 \%$ are because of the Roche lobe effect (the difference between the $0.3 M_{\odot}$ and the $1.0 M_{\odot}$ curve for $\mathrm{G}$ star radiation denoted RO). From this consideration it can be seen that Roche lobe effects are important, but the different radiation regimes give the main contribution for the different loss rates of Neptune-mass planets.

For Jupiter-mass planets the absolute loss is much smaller, so the involved processes are also less pronounced. However, the Roche lobe effect is rather important, just because the relative radiation-induced mass loss is small. At planets with the mass of Saturn, the effects of radiation and Roche lobe effects make a similar contribution to the total mass loss.

\subsection{Final planetary mass distributions for an initial flat mass distribution}

As in Penz et al. (2008) we assume a flat distribution of masses ranging from $0.2 M_{\text {nep }}$ up to $10 M_{\text {jup }}$. The resulting distribution functions after $4 \mathrm{Gyr}$ are plotted in Fig. 7. For the close-in orbit of $0.02 \mathrm{AU}, 11 \%$, and $2 \%$ of the planets are lost, for a density of 0.4 and $1.4 \mathrm{~g} / \mathrm{cm}^{3}$, respectively. At a distance of $0.05 \mathrm{AU}$, none of the planets are lost. Due to the mass loss the final distribution functions are shifted to lower masses compared with the initially flat distribution. If we compare these mass distributions with the one for dG stars we can see that Jupiter-mass planets are less effected and the distribution function in this mass range is similar to the initial distribution for $\mathrm{dM}$ stars. In the low-mass tail, fewer planets are found around dG stars because of the higher radiation emitted by these stars. 


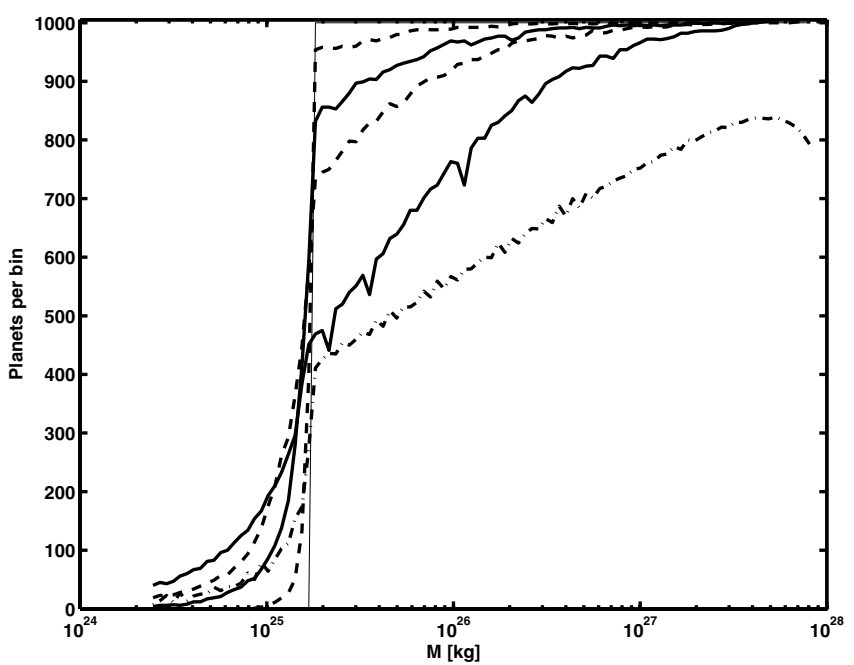

Fig. 7. Initial flat mass distribution (thin vertical line) and final mass distributions after 4 Gyr for 0.4 (solid lines) and $1.4 \mathrm{~g} / \mathrm{cm}^{3}$ (dashed lines). Orbital distances are 0.02 and $0.05 \mathrm{AU}$ (starting from the lower curve). As comparison, the mass distribution for $\mathrm{dG}$ stars at $0.02 \mathrm{AU}$ and for $0.4 \mathrm{~g} / \mathrm{cm}^{3}$ is shown (dashed-dotted line; from Penz et al. 2008).

\section{Loss history of GJ 876d and GJ 674b}

The most challenging known planet to prove theories about atmospheric evaporation is GJ 876d (Rivera et al. 2005), which is the innermost planet in a triple system with two Jupiter-mass planets. It has a mass of $0.024 M_{\text {jup }}$ and is orbiting at a distance of $0.021 \mathrm{AU}$. Its host star is an M star with a mass of $0.32 M_{\odot}$ and an estimated age between 6.5 and 9.9 Gyr (Saffe et al. 2005, and references therein). The X-ray luminosity of GJ 876 is given by Schmitt \& Liefke (2004) with $10^{26.49} \mathrm{erg} / \mathrm{s}$. It can be seen in Fig. 2 that it is a quiet star with an X-ray luminosity below the median value at its age. We use the scaling law derived in this paper to discuss possible evolutionary scenarios for GJ 876d. Since the density of this planet is unknown, we use a range from $0.1-10 \mathrm{~g} / \mathrm{cm}^{3}$ and assume an age of the system of $10 \mathrm{Gyr}$. Now we can calculate the starting mass of the planet at $0.1 \mathrm{Gyr}$, which is shown in Fig. 8. One can see that, only for very low densities of $<0.1 \mathrm{~g} / \mathrm{cm}^{3}$, the starting mass of the planet has to be about $4 \times 10^{26} \mathrm{~g}\left(\sim 0.2 M_{\text {jup }}\right)$, which is still below the mass of Saturn. For densities above $1 \mathrm{~g} / \mathrm{cm}^{3}$, no significant change in the mass is caused by $\mathrm{X}$-ray radiation.

To calculate the survival probability of such a planet, we assume that the planet had an initial mass of $0.024 M_{\text {jup }}$ and calculate how such a planet would evolve. This is shown in Fig. 9, where it can be seen that for low densities up to about $0.5 \mathrm{~g} / \mathrm{cm}^{3}$ the planet can be evaporated. For densities up to $2 \mathrm{~g} / \mathrm{cm}^{3}$, significant parts of the atmosphere can be lost, but the planet can survive. For higher densities the change in the planetary mass is negligible. These results are different from the results obtained by Lecavelier des Etangs (2007), who concluded that GJ 876d must have at least a density higher than $3.1 \mathrm{~g} / \mathrm{cm}^{3}$ to survive over 5 Gyr. The reason for this disagreement is mainly based on the different radiation input used in this work. Lecavelier des Etangs (2007) uses a median value for the XUV luminosity of dM stars derived by Hodgkin \& Pye (1994) and scales this value using the present-day solar EUV luminosity. This approach relies on the median value given by Hodgkin \& Pye (1994), which is based on a detection rate of only $10 \%$; therefore the quoted median value, computed with a maximum likelyhood technique, is indeed an upper limit to the true median value. Additionally, it assumes

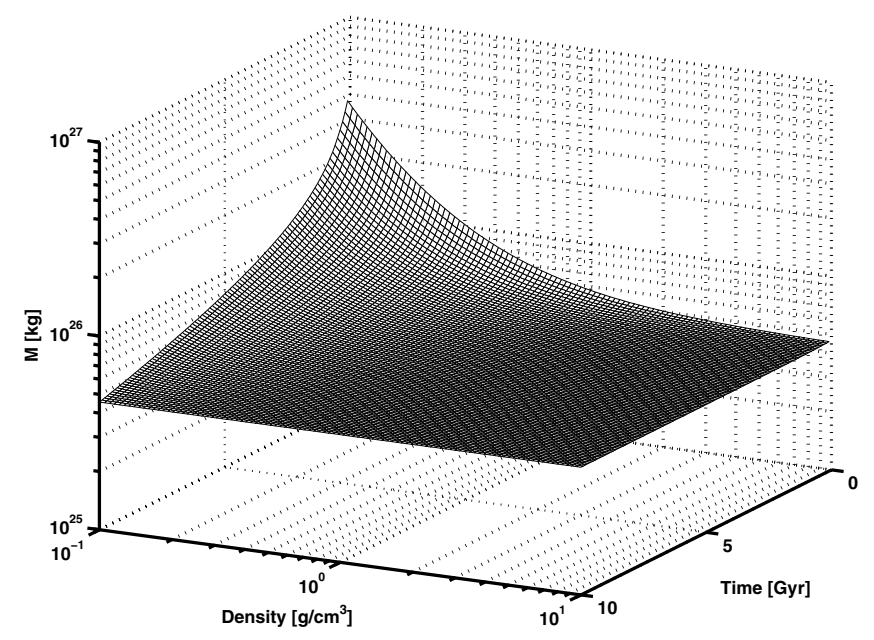

Fig. 8. The mass evolution of GJ 876d over $10 \mathrm{Gyr}$ for densities from $0.1-10 \mathrm{~g} / \mathrm{cm}^{3}$ to reach a final mass of $0.024 M_{\text {jup }}$.

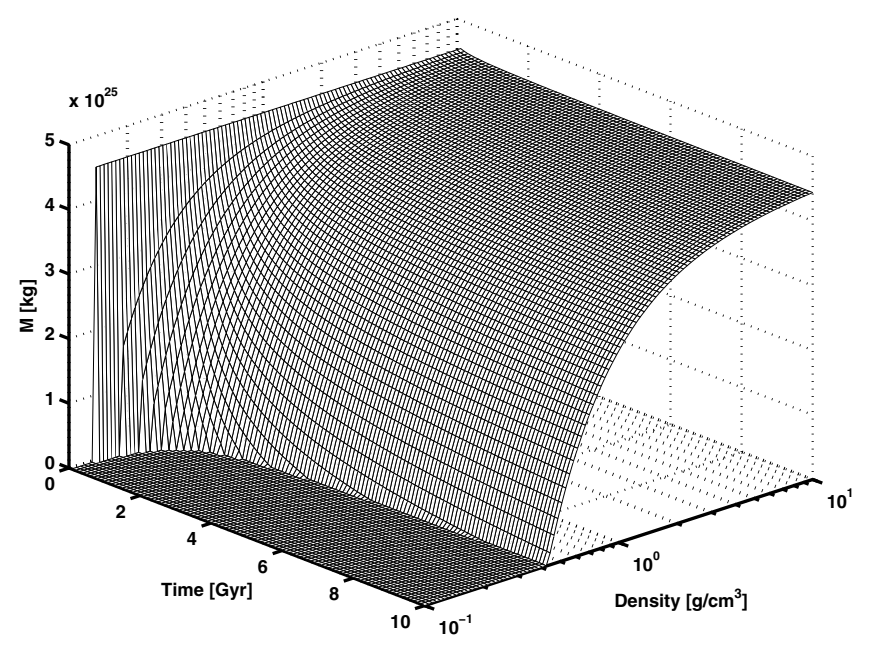

Fig. 9. The mass evolution of GJ 876d over $10 \mathrm{Gyr}$ for densities from $0.1-10 \mathrm{~g} / \mathrm{cm}^{3}$ using an initial mass of $0.024 M_{\text {jup }}$.

that the correlation for the S2 luminosities between $\mathrm{dM}$ and $\mathrm{dG}$ stars is valid for the whole wavelength range from 1-1180 $\AA$ flux, for which the solar EUV luminosity is derived. Using the value of $2.9 \mathrm{erg} \mathrm{cm}^{-2} \mathrm{~s}^{-1}$ given by Lecavelier des Etangs (2007) for dM stars, a median EUV luminosity of $10^{27.91} \mathrm{erg} / \mathrm{s}$ is derived for 4.6 Gyr. Using the scaling law for the radiation evolution following $t^{-1}$ as proposed by Lecavelier des Etangs (2007), the EUV luminosity of GJ 876 at an age of $10 \mathrm{Gyr}$ is $10^{27.57} \mathrm{erg} / \mathrm{s}$, while we used the observed X-ray luminosity of $10^{26.49} \mathrm{erg} / \mathrm{s}$ (Schmitt \& Liefke 2004). This different treatment of the radiation input explains the different conclusions drawn in these works. The results presented in our paper should be considered as a lower limit since we only investigate the contribution of $\mathrm{X}$-rays. Unfortunately, this precludes a definitive conclusion on the nature of GJ876d.

From Fig. 2 it is obvious that GJ 674 is also a quiet star, even if younger. Its age is ranging from 0.1-1 Gyr (Bonfils et al. 2007) and the X-ray luminosity given in Schmitt \& Liefke (2004) is $10^{27.6} \mathrm{erg} / \mathrm{s}$. Assuming an age of $1 \mathrm{Gyr}$, we can calculate the initial mass of its companion, whose present mass is given as $0.037 M_{\text {jup }}$ and whose orbital distance is $0.039 \mathrm{AU}$ (Bonfils et al. 2007). From Fig. 10 it can be seen that even for unrealistic densities of $0.1 \mathrm{~g} / \mathrm{cm}^{3}$, the initial mass was 


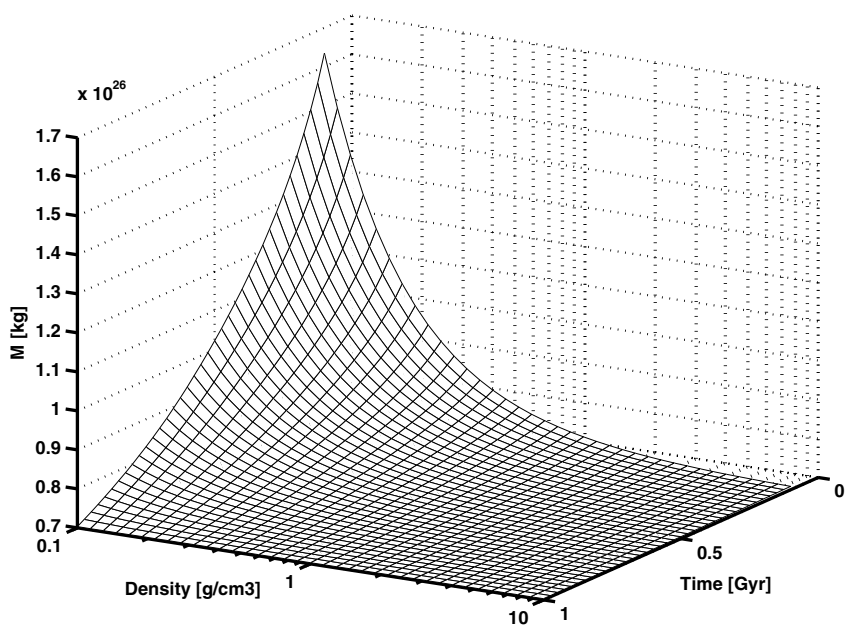

Fig. 10. The mass evolution of GJ 674b over $1 \mathrm{Gyr}$ for densities from $0.1-10 \mathrm{~g} / \mathrm{cm}^{3}$ to reach a final mass of $0.037 M_{\text {jup }}$.

about $1.7 \times 10^{26} \mathrm{~g}\left(\sim 0.1 M_{\text {jup }}\right)$. For densities around $1 \mathrm{~g} / \mathrm{cm}^{3}$, the mass loss is already negligible. In the case of this exoplanet, it is very unlikely that it evolved from a higher mass planet due to thermal mass loss effects to its present mass.

\section{Conclusions}

In this work we investigate the influence of the X-ray luminosity distribution of $\mathrm{dM}$ stars on the mass loss from planets orbiting at close-in distances. The X-ray flux emitted by dM stars is smaller than for $\mathrm{dG}$ stars, therefore planets with the same parameters are less affected by mass loss processes if they are orbiting an M dwarf. We find that Jupiter-mass planets cannot be completely evaporated, even if they have a low density of $0.4 \mathrm{~g} / \mathrm{cm}^{3}$ after 4 Gyr. For Neptune-mass planets with a density of $0.8 \mathrm{~g} / \mathrm{cm}^{3}$, $10 \%$ of the planets might be evaporated or eroded to its dense core, while the remaining $90 \%$ of the planets are able to keep an $\mathrm{H} / \mathrm{He}$ envelope or even remain hydrogen-rich gas planets over their lifetime. Splitting up the Roche lobe and the radiation effect, we show that the radiation effect has a stronger effect on Neptune-mass planets, while the Roche lobe effect becomes relatively strong for Hot Jupiters, where radiation-induced mass loss is small. An initially flat mass distribution for planets with masses ranging from $0.2 M_{\text {nep }}$ to $10 M_{\text {jup }}$ is mainly influenced at masses below $1 M_{\text {jup }}$, and at a distance of $0.02 \mathrm{AU}, 11 \%$ of the low-density planets are lost. Finally, we investigate the mass loss for GJ 876d and GJ 674b. Both are orbiting quiet stars, and for planetary densities $>1 \mathrm{~g} / \mathrm{cm}^{3}$, no significant change in the mass is caused by the incoming $\mathrm{X}$-ray radiation.

The presented work is subject to several simplifications because of the limited data available, discussed in detail in Penz et al. (2008). The scaling law used for the density is a simplified approach and cannot be applied to planets with masses lower than Neptunes. Further modelling and observations should help to overcome this problem. The assumed heating efficiency of $100 \%$ is another simplification, which might be resolved by detailed radiative transfer calculations. The derived scaling law for the X-ray luminosity is based on a few points, and especially for ages $>600 \mathrm{Myr}$ it is not possible to gather information about luminosity distributions of older clusters with the current instrumentation. However, this assumption is not cruciallinebreak since most of the effects occur at a young age. Also the EUV flux from 200-900 , where we have no information because of strong interstellar absorption, should be investigated and added in future.

Acknowledgements. This work is supported by the Marie Curie Fellowship Contract No. MTKD-CT-2004-002769 of the project "The influence of stellar high radiation on planetary atmospheres", and the host institution INAFOsservatorio Astronomico di Palermo. Also acknowledged is support by ASI-INAF contract I/088/06/0.

\section{References}

Adams, E. R., Seager, S., \& Elkins-Tanton, L. 2007, ApJ, accepted [arXiv: 0710.4941]

Baraffe, I., Selsis, F., Chabrier, G., et al. 2004, A\&A, 419, L13

Baraffe, I., Chabrier, G., Barman, T. S., et al. 2005, A\&A, 436, L47 Bonfils, X., Forveille, T., Delfosse, X., et al. 2005, A\&A, 443, L15 Bonfils, X., Mayor, M., Delfosse, X., et al. 2007, A\&A, 474, 293 Butler, R. P., Vogt, S. S., Marcy, G. W., et al. 2004, ApJ, 617, 580 Butler, R. P., Wright, J. T., Marcy, G. W., et al. 2006, ApJ, 646, 505 Cecchi-Pestellini, C., Ciaravella, A., \& Micela, G. 2006, A\&A, 458, L13 Cox, A. N. 2001, Allen's astrophysical quantities (Springer)

Demory, B.-O., Gillon, M., Barman, T., et al. 2007, A\&A, submitted [arXiv: 0707.3809]

Dorren, J. D., \& Guinan, E. F. 1994, in The Sun as a Variable Star: Solar and Stellar Irradiance Variations, ed. J. M. Pap, C. Frolich, H. S. Hudson, \& S. Solanki, Proc. IAU Colloq., 143, 206

Eggenberger, P., Charbonnel, C., Talon, S., et al. 2004, A\&A, 417, 235

Erkaev, N. V., Lammer, H., Kulikov, Yu. N., et al. 2007, A\&A, 472, 329 Garcia Muñoz, A. 2007, Planet. Space Sci., 55, 1426

Gillon, M., Pont, F., Demory, B.-O., et al. 2007, A\&A, 472, L13

Guinan, E. F., Engle, S. G., Ribas, I., Schulze-Makuch, D., \& McCook, G. P. 2007, Am. Astron. Soc. Meet. Abstracts, 210, \# 33.03

Hodgkin, S. T., \& Pye, J. P. 1994, MNRAS, 267, 840

Hubbard, W. B., Hattori, M. F., Burrows, A., Hubeny, I., \& Sudarsky, D. 2006, Icarus, 187, 358.

Hubbard, W. B., Hattori, M. F., Burrows, A., \& Hubeny, I. 2007, ApJ, 658, L59 Ida, S., \& Lin, D. N. C. 2005, Prog. Theor. Phys. Suppl., 158, 68

Johnson, J. A., Butler, R. P., Marcy, G. W., et al. 2007, ApJ, accepted [arXiv: 0707.2409]

Lada, C. J., Muench, A. A., Luhmann, K. L., et al. 2006, AJ, 131, 1574

Laughlin, G., Bodenheimer, P., \& Adams, F. C. 2004, ApJL, 612, 73

Lammer, H., Selsis, F., Ribas, I., Guinan, E. F., \& Bauer, S. J. 2003, ApJ, 598, L121

Lammer, H., Lichtenegger, H. I. M., Kulikov, Yu. N., et al. 2007, Astrobiology, 7,185

Lecavelier des Etangs, A. 2007, A\&A, 461, 1185

Lecavelier des Etangs, A., Vidal-Madjar, A., McConnell, J. C., \& Hébrard, G. 2004, A\&A, 418, L1

Liu, M. C., Matthews, B. C., Williams, J. P., \& Kalas, P. G. 2004, ApJ, 608, 526 Marcy, G. W., Butler, R. P., Vogt, S. S., Fischer, D., \& Lissauer, J. J. 1998, ApJ, 505, L147

Miglio, A., \& Montalbán, J. 2005, A\&A, 441, 615

Penz, T., Micela, G., \& Lammer, H. 2008, A\&A, 477, 309

Pizzolato, M., Maggio, A., Micela, G., Sciortino, S., \& Ventura, P. 2003, A\&A, 397, 147

Preibisch, T., \& Feigelson, E. D. 2005, ApJS, 160, 390

Ribas, I., Guinan, E. F., Güdel, M., \& Audard, M. 2005, ApJ, 622, 680

Rivera, E. J., Lissauer, J. J., Butler, R. P., et al. 2005, ApJ, 634, 625

Saffe, C., Gómez, M., \& Chavero, C. 2005, A\&A, 443, 609

Scalo, J., Kaltenegger, L., Segura, A., et al. 2007, Astrobiology, 7, 85

Schmitt, J. H. M. M., \& Liefke, C. 2004, A\&A, 417, 651

Schmitt, J. H. M. M., Fleming, T. A., \& Giampapa, M. S. 1995, ApJ, 450, 392

Selsis, F., Kasting, J. F., Levrard, B., et al. 2007, A\&A, 476, 1373

Stauffer, J. R., Rebull, L. M., Carpenter, J., et al. 2005, ApJ, 130, 1834

Stelzer, B., \& Neuhäuser, R. 2001, A\&A, 377, 538

Tian, F., Toon, O. B., Pavlov, A. A., \& De Sterck, H. 2005, ApJ, 621, 1049

Udry, S., Bonfils, X., Delfosse, X., et al. 2007, A\&A, 469, L43

Vilhu, O., \& Walter, F. M. 1987, A\&A, 321, 958

Watson, A. J., Donahue, T. M., \& Walker, J. C. G. 1981, Icarus, 48, 150

Yelle, R. 2004, Icarus, 170, 167

Yelle, R. 2006, Icarus, 183, 508 